\title{
Effects of pethidine and its antagonists on the newborn
}

\author{
J. E. H. BRICE, T. A. MORELAND, AND C. H. M. WALKER
}

Departments of Child Health, Pharmacology and Therapeutics, Ninewells Hospital and Medical School, Dundee

SUMMARY Nalorphine and naloxone were compared as to their effectiveness as pethidine antagonists. 85 infants were divided into a control group comprising 19 newborn babies whose mothers did not receive pethidine and the babies received no antagonist, and three groups in which the mothers all received pethidine and the babies had either no antagonist (24), nalorphine IV (16), or naloxone IV (26). All the babies were assessed by measuring their neurobehavioural states and respiratory functions. A further 12 newborn babies had naloxone plasma levels measured by radioimmunoassay. Although standard doses of nalorphine effectively antagonised the depressive effect on respiration induced by pethidine, there was a pronounced and undesirable excitatory agonist action. Naloxone was not observed to have any agonist activity, but the recommended IV dose $(0.01 \mathrm{mg} / \mathrm{kg})$ had only a slight and delayed antagonist action as measured by respiratory function tests. A more rapid and improved antagonism was noted after this dose was doubled $(0.02 \mathrm{mg} / \mathrm{kg})$. The plasma elimination-phase half-life of naloxone after intravenous cord injection was about 3 hours.

The narcotic antagonist naloxone became available in the UK in 1976, but there are few reports of its use in newborn babies. Naloxone can restore respiratory function to normal after depression induced by pethidine (Evans et al., 1976; Gerhardt et al., 1977; Wiener et al., 1977). Studies in animals and man have shown that the main advantage of naloxone over nalorphine is its freedom from agonist activity, while a disadvantage is that its effect after IV injection seems to be of short duration. However, Wiener et al. (1977) showed this effect could be prolonged to 24-48 hours or longer by a single, large IM injection. These workers also demonstrated the ability of naloxone to restore neurobehavioural function in the newborn babies of mothers who had received pethidine.

Nalorphine was the standard narcotic antagonist used in the newborn until the introduction of naloxone, but their antogonist activities have not been compared. This study was therefore designed to compare the action of these two drugs, to assess the

Neonatal Unit, City Hospital, Nottingham

J. E. H. BRICE, senior registrar

Ninewells Hospital and Medical School, Dundee

Department of Child Health

C. H. M. WALKER, senior lecturer

Department of Pharmacology and Therapeutics

T. A. MORELAND, lecturer effect of currently recommended doses of naloxone, and to determine its plasma levels and half-life in the newborn baby.

\section{Methods}

Informed written consent for the study was obtained from the parents before labour was established. Only babies born at term (37-42 weeks) after a normal pregnancy, labour, and delivery were studied; babies with a cord blood $\mathrm{pH}$ of $<7 \cdot 25$, babies who did not respond to simple resuscitative measures, and babies with any significant abnormalities were excluded. 85 newborn babies were divided at random into four groups. In the control group (group 1, $\mathrm{n}=$ 19) the mothers had received no pethidine during labour and their babies received no antagonist. A few mothers received epidural analgesia and/or Entonox, but no other analgesic or sedative was used. In the other groups the mothers had received IM pethidine within 4 hours of delivery but no other analgesic or sedative, apart from Entonox. Treatment of the newborn was randomised so that group 2 $(n=24)$ received no narcotic antagonist, and group $3(n=16)$ received nalophine and group 4 $(n=26)$ naloxone between one and 2 minutes after delivery. The antagonist was injected directly into the cord vein as close to the umbilicus as 
possible and 'milked' inwards before the cord was reclamped at the site of injection. Apart from 3 newborn babies who received $0.7 \mathrm{mg}$ nalorphine at the start of the study, the dose of this drug was standardised at $0.5 \mathrm{mg}$. Group 4 were divided into two different dose regimens: the recommended IV dose $(0.01 \mathrm{mg} / \mathrm{kg} \simeq 0.035 \mathrm{mg})$ was given to 14 babies in group 4A and, after evaluating the results of this treatment, 12 babies (group 4B) were given double this dose $(0.07 \mathrm{mg})$.

The newborn babies were assessed clinically in three ways. The wakefulness of each was recorded at 15,30 , and 90 minutes and again between 12 and 24 hours, using the six states described by Brazelton (1973). At 90 minutes and again between 12 and 24 hours the sucking reflex was assessed, using the method of Scanlon et al. (1974), and between 12 and 24 hours of age a Scanlon neurobehavioural score was performed by two paediatric registrars who were unaware of the treatment the mother and baby had received.

As part of the respiratory function assessment, the times from delivery to the 1st breath and to sustained respiration were noted, together with the routine recording of Apgar scores. During the definitive tests of respiratory function the babies were nursed in a heated cot in which the skin temperatures were maintained within the neutral thermal range $\left(36 \cdot 0-36 \cdot 5^{\circ} \mathrm{C}\right)$ by servo-control. If possible the measurements were performed with the newborn in a stable state, although this was difficult in group 3. The main test of respiratory function was the measurement of carbon dioxide $\left(\mathrm{CO}_{2}\right)$ output, from which, together with heel prick arterialised capillary blood $\mathrm{pCO}_{2}$, alveolar ventilation was calculated (alveolar ventilation $=$ expired $\mathrm{CO}_{2} \times$ barometric pressure $/ \mathrm{pCO}_{2}$, corrected for temperature and humidity). These estimations and full blood-gas analyses were done at 15,30 , and 90 minutes and finally between 12 and 24 hours after delivery. Carbon dioxide output was determined using a technique derived from open-circuit methods previously described (Kappagoda et al., 1974; Newell et al., 1976; Evans et al., 1977). Expired $\mathrm{CO}_{2}$ was measured using an infrared gas analyser*, and the overall error of the calculated expired carbon dioxide output with this system was less than $\pm 5 \%$ (Thompson et al., 1979). Statistical evaluation was done with Student's $t$ test.

Heel prick blood samples were collected from a separate group of 12 newborn babies for determination of plasma naloxone levels by radioimmunoassay. It was necessary to use different babies because sampling disturbed the baby sufficiently to invalidate the respiratory function result as representing the 'basal' state. Half of them had received $0.035 \mathrm{mg}$ and half $0.07 \mathrm{mg}$ naloxone injected into the umbilical vein. The assay was such that $2 \mathrm{ng}$ naloxone displaced the radiolabelled tracer, ${ }^{3} \mathrm{H}$-naloxone, by $50 \%$. The presence of pethidine in concentrations of up to $\mathbf{4 0 0}$ ng per sample caused no displacement. Each assay was carried out in duplicate with $0.2 \mathrm{ml}$ heparinised plasma. Although the main metabolites of naloxone were not available to test for crossreactivity, current evidence suggests that they would be unlikely to interfere with the assay in the concentrations likely to be found in plasma (Berkowitz et al., 1975).

\section{Results}

The items of clinical relevance are shown in Table 1. The mean maternal pethidine doses and the mean pethidine-to-delivery time intervals of the three groups of mothers receiving pethidine were not significantly different. Despite randomisation the difference between the mean birthweights of the no antagonist babies and those in group 3 was significant $(P<0.05)$, but when compared with the mean birthweights of the other groups this was probably not of clinical importance. That the newborn had not been subject to intrauterine hypoxia is suggested by the normal cord blood $\mathrm{pH}$ findings, the slight differences

*Analytical Development Co. Ltd. type 901 ; P. K. Morgan Ltd.

Table 1 Mean $( \pm S D)$ of maternal pethidine dose, pethidine-delivery interval, birthweight, cord blood pH, Apgar score, and time to sustained respiration

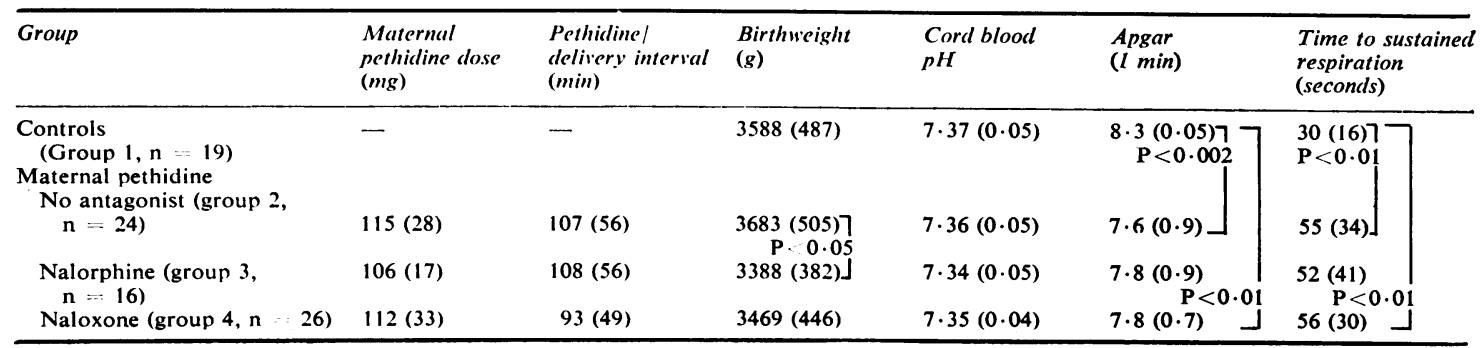


between the groups being of no significance. There was, however, evidence of pethidine intoxication as reflected in the Apgar score, the mean one-minute value of 7.6 for the no-antagonist group being lower than the $8 \cdot 3$ for the control group $(P<0 \cdot 002)$. Likewise, failure to establish respiration normally was seen in the 3 pethidine-treated groups in that the time to sustained respiration was prolonged (52-56 seconds) when compared with the control group ( 30 seconds, $P<0 \cdot 01$ ). Groups 3 and 4 showed a similar initial degree of respiratory depression, but because of the smaller numbers the differences were not all of statistical significance.

The Brazelton state score indicated that the newborn babies in all groups were in a rather wakeful condition for the first 90 minutes (range of mean scores $3 / 6$ to $5 / 6$ ) but that they had settled by the 12-24-hour assessment (range 1.7/6-1.9/6). Infants in group 3 tended to be fretful and irritable with high pitched cries, and their scores were appropriately high (range $5 / 6-6 / 6$ at 15 and 30 minutes). This was associated with a poor mean Scanlon sucking score at 90 minutes of $1.0 / 3$ compared with $>1.9 / 3$ for the other groups. By 12-24 hours there was a difference in the sucking scores between the controls $(1 \cdot 3 / 3)$ and group 3 $(1 \cdot 8 / 3)$, but there was no obvious difference between the no-antagonist and the antagonist-treated groups. There was no significant difference in the mean and decrement values for the Scanlan and neuro- behavioural scores (group 1, 18.00 $\pm 6 \cdot 18$ and $18 \cdot 33 \pm 6 \cdot 93$; group $2,19 \cdot 23 \pm 3.98$ and $15 \cdot 31 \pm$ $6 \cdot 29$; group $3,18.57 \pm 4.22$ and $14.47 \pm 8.61$; group $4 A$ and $B, 18 \cdot 07 \pm 4 \cdot 46$ and $17 \cdot 5 \pm 5 \cdot 26$ ).

Multiple statistical comparisons were made between the mean $\mathrm{pH}, \mathrm{pO}_{2}, \mathrm{pCO}_{2}$, and base deficit measurements from each group. Many of the differences were statistically significant (e.g. $\mathrm{pH}$ at 15 and 90 minutes between group 1 and $2, P<0.001$; $\mathrm{pH}$ at 15 minutes between group 1 and group 4A, $P<0.05$, and group 4B, $P<0.001$ ). The results show that there was respiratory depression in group 2 and even in group 4A. Babies in group 4B fared rather better, and these trends are in keeping with the results of the specific respiratory function studies that follow.

Tables 2 and 3 show the values for total expired carbon dioxide $\left(\mathrm{CO}_{2}\right.$ output $)$ and alveolar ventilation in the four groups. At all times group 2 showed a consistent reduction in both these parameters compared with group $1\left(\mathrm{P}<0.01\right.$ for $\mathrm{CO}_{2}$ output at 15 and 30 minutes and alveolar ventilation at 15 minutes). By 12-24 hours the difference in $\mathrm{CO}_{2}$ output was less significant $(P<0.02)$ and the differences in alveolar ventilation were no longer significant.

In group 3 the values for $\mathrm{CO}_{2}$ output and alveolar ventilation were consistently higher at 15,30 , and 90 minutes than those of group 1 . They were also significantly higher than those of group 2 at 15, 30,

Table 2 Mean $( \pm S D)$ of expired $\mathrm{CO}_{2}$ output ( $\mathrm{ml} / \mathrm{kg}$ per min BTPS)

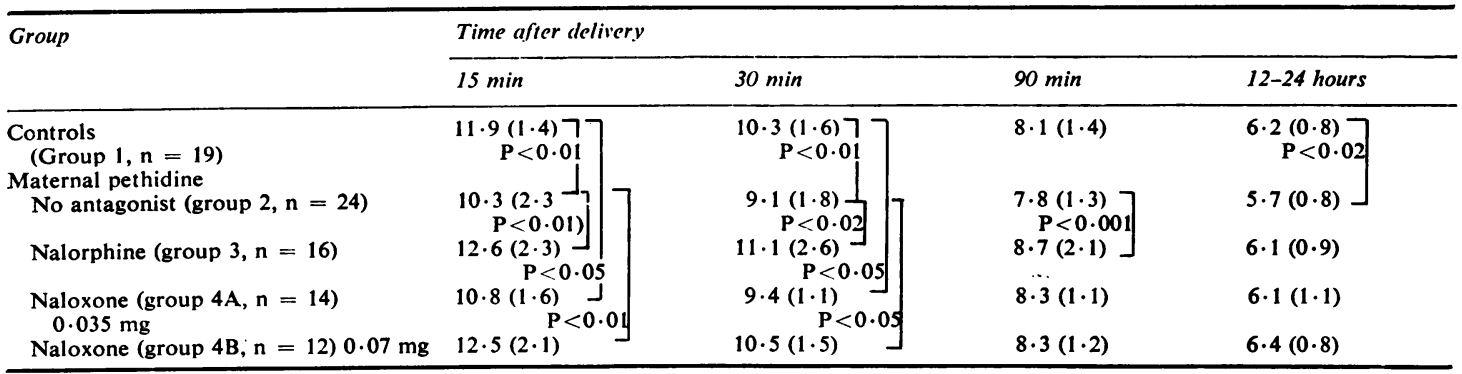

Table 3 Mean $( \pm S D)$ of alveolar ventilation $(\mathrm{ml} / \mathrm{kg} / \mathrm{per} \min \mathrm{BTPS})$

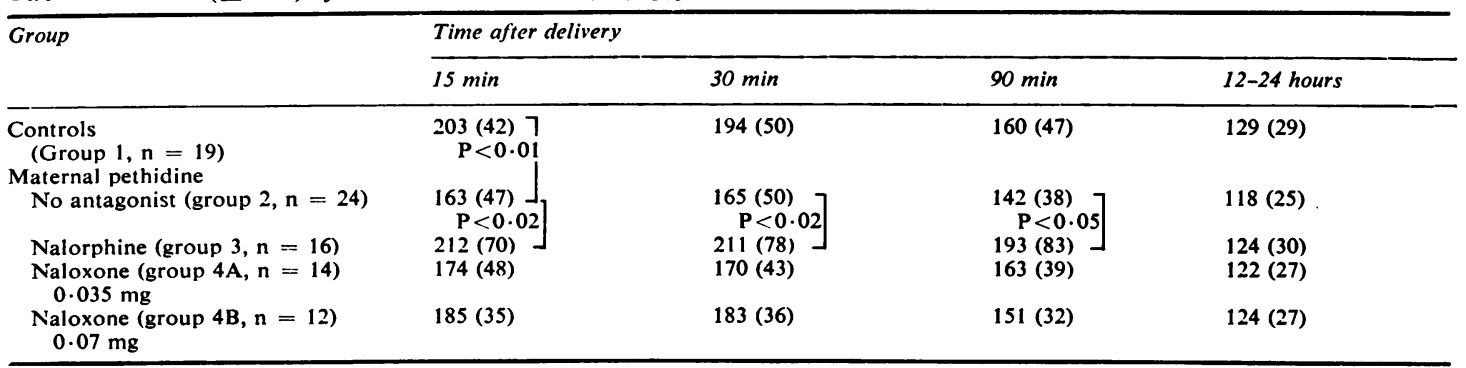


and 90 minutes with a smaller nonsignificant difference at 12-24 hours. Although the former were not statistically significant they do reflect a state of hyperventilation in group 3 compared with both the groups 1 and 2, and this was also manifest in a reduced capillary $\mathrm{pCO}_{2}$ at 90 minutes.

After the lower naloxone dose in group $4 \mathrm{~A}, \mathrm{CO}_{2}$ output and alveolar ventilation at 15 and 30 minutes were similar to those in group 2 , indicating inadequate antagonism, and there was a significant reduction in $\mathrm{CO}_{2}$ output $(\mathrm{P}<0.05)$ in this group compared with the controls (group 1). However, there appeared to be a delayed naloxone effect with alleviation of respiratory depression at 90 minutes, the lack of statistical significance in this trend being due perhaps to the small number of cases.

In group 4B there was an improved initial antagonism. However, the values for alveolar ventilation (and capillary blood-gases) suggest that reversal of respiratory depression was not complete (Table 3). Compared with group 2, the $\mathrm{CO}_{2}$ output was significantly higher at 15 minutes $(\mathrm{P}<0.01)$ and at 30 minutes $(P<0.05)$ (Table 2). By 90 minutes the $\mathrm{CO}_{2}$ output differences between groups 1,2 , and 4B were no longer significant but the values for both $\mathrm{CO}_{2}$ output and alveolar ventilation suggested continuing antagonism.

In groups 2,3 , and 4 the alveolar ventilation values were correlated against the pethidine-todelivery intervals of $0-1,1-2,2-3$, and 3-4 hours. While there was obviously less respiratory depression in the 0-1-hour interval group, the differences were not significant, probably again due to the few infants in each group.

The results for the serial blood-gas analyses, the Brazelton and Scanlon scores, and the relation of alveolar ventilation to the pethidine-to-delivery intervals are not presented in full.*

The radioimmunoassay method was sensitive enough to enable naloxone levels to be followed for up to 4 hours after drug administration (Moreland et al., 1979). Peak plasma levels of 4.0 to $20 \cdot 2 \mathrm{ng} / \mathrm{ml}$ (mean 11.0) were observed during the first 40 minutes. Concentrations at the end of 4 hours ranged from 1.4 to $6.6 \mathrm{ng} / \mathrm{ml}$ (mean $4 \cdot 2$ ). In 4 newborn babies the drug concentration fell rapidly during the first 40 minutes and then declined more slowly, with an initial (distribution-phase) half-life calculated in one baby of 8 minutes. No such distribution phase was observed in the others and in 4 babies the plasma drug concentration increased during the first 20 to $\mathbf{4 0}$ minutes. The eliminationphase (mean \pm SD) half-life calculated from all 12 newborn babies was $185 \pm 108 \mathrm{~min}$. The apparent

\footnotetext{
*These may be obtained from J.E.H.B. on request.
}

volume of distribution determined by extrapolating the elimination phase curve was $1.99 \pm 0.28 \mathrm{l} / \mathrm{kg}$ and the plasma clearance rate was $0.57 \pm 0.121 / \mathrm{kg}$ per hour. There was no significant difference in the half-life, apparent volume of distribution, or clearance values between the newborn given $0.035 \mathrm{mg}$ and $0.07 \mathrm{mg}$ naloxone.

\section{Discussion}

The fact that relatively few cases were suitable for study during the 18 months of this project was due to the very strict criteria for selection, every effort having been made to eliminate all newborn babies with other depressant and complicating perinatal factors. During the study over $60 \%$ of the mothers received pethidine analgesia at some stage. The total dose received was relatively small and it was rare for a mother to be given a second dose, epidural anaesthesia being used if necessary. Despite this frequent but careful use of pethidine, our results confirm that significant depression of respiratory function was produced in these babies and, although slight, the differences were still apparent 12-24 hours after delivery. The fact that the effects of pethidine can be detected for even longer has been confirmed by Cooper et al. (1977) who found that it may take the newborn 3 to 6 days finally to eliminate the drug. Although not presented in detail, the respiratory function results were divided according to the pethidine-to-delivery time interval, and more obvious respiratory depression was found when the pethidine had been administered more than one hour before delivery.

Clinical assessments tend to be subjective and our preliminary observations of the state of wakefulness and of the sucking reflex were not performed under double-blind conditions. However, the pronounced excitatory effect of nalorphine was obvious and this confirms the clinical impression of hyperexcitability held previously. The Scanlon behavioural score, performed at 12-24 hours under double-blind conditions, indicated that there were no differences between the four groups. This differs from the findings of Brackbill et al. (1974), and may be due to the small numbers, to the timing, or to the subjective nature of the test. More objective tests such as those adopted by Wiener et al. (1977) to assess feeding, sucking, and habituation to an auditory stimulus are probably more accurate and reliable.

Nalorphine, although an effective antagonist to pethidine in terms of restoration of respiratory function, had an obvious excitatory effect, although it was not possible to estimate how much of the improvement was due to the narcotic antagonism and how much to stimulant effects. The most 
pronounced excitatory evidence of nalorphine was seen in the babies whose mothers had received pethidine within one hour of delivery, the group in which the babies were less likely to be depressed. No evidence of depressant effects of nalorphine were observed.

Since this study was completed nalorphine has been withdrawn from the market, so naloxone is now the only neonatal narcotic antagonist. As expected, no agonist activity of naloxone in the two doses used was observed. However, the results suggest that the recommended IV dose $(0.01 \mathrm{mg} / \mathrm{kg})$ when given as a bolus injection into the cord is ineffective at 15 and 30 minutes in reversing pethidine-induced respiratory depression. The apparent delayed effect (at 90 minutes) of the smaller dose could be a random finding related to the fewer cases. A more probable explanation, and one compatible with data on naloxone plasma levels, is that the drug is retained in the umbilical vein and ductus venosus, so delaying the distribution into the systemic circulation.

An improved and more rapid effect was seen with the larger $(0.02 \mathrm{mg} / \mathrm{kg})$ dose which may have been flushed more rapidly into the systemic circulation because of the larger volume. The purpose of this work was to study the antagonists in the circumstances most likely to be found in everyday obstetric practice, so the effects of umbilical catheter or peripheral IV injection, which have practical disadvantages when dealing with the acutely depressed newborn, were not studied.

Evans et al. (1976) used alveolar $\mathrm{pCO}_{2}$ in the calculation of alveolar ventilation, while we used capillary $\mathrm{pCO}_{2}$ and it might be expected that the values in the two studies would differ slightly. Nevertheless, allowing for such differences in method, the general conclusions about the effects of IV naloxone are similar. Evans et al. (1976) and Wiener et al. (1977) studied IV naloxone at one dose only $(0.04 \mathrm{mg})$ and our results support their finding that the effect of such a dose is transient, and they even suggest that the dose can safely be doubled. The results of a large IM dose of naloxone $(0.2 \mathrm{mg})$ (Wiener et al., 1977) are impressive, and demonstrate a prolonged effect. The values for $\mathrm{CO}_{2}$ output $(7 \cdot 1-7.5 \mathrm{ml} / \mathrm{min}$ per $\mathrm{kg})$ and alveolar ventilation $(190.0-194.4 \mathrm{ml} / \mathrm{min}$ per $\mathrm{kg})$ at $12-48$ hours are very high compared with our own control values at 12-24 hours and with other reported figures (Nelson et al., 1962). A large dose of naloxone may have an effect on respiratory function other than purely narcotic antagonism. The findings almost suggest that this massive dose is exerting an excitatory effect similar to that found in nalorphine-treated babies. This explanation is supported by the work of Bramwell and Bradley (1974) in which both nalorphine and naloxone were found to exert an effect at the locus for the excitatory effects of morphine. Whether levels of naloxone rise sufficiently rapidly after IM injection to obviate the need for an IV dose in the acutely depressed and asphyxiated newborn baby is still uncertain. Possibly the acutely depressed baby should be given an IV dose followed by a larger IM one.

The elimination-phase plasma half-life of naloxone in the newborn ( 3 hours) was considerably longer than that in the adult (one hour) (Ngai et al., 1976). This is probably due to immaturity of the drugmetabolising enzyme systems in the newborn. This reduced rate of elimination after comparable dosage would be expected to lead to longer duration of action of naloxone in the infant compared with the adult, longer than the 30 minutes suggested previously (Evans et al., 1976; Wiener et al., 1977).

We confirm that large IV doses of naloxone, unlike nalorphine, do not have any obvious narcotic agonist activity in the newborn child. To produce rapid and effective narcotic antagonism, the recommended IV dose of naloxone could be safely doubled to $0.02 \mathrm{mg} / \mathrm{kg}$ and perhaps increased even more. As respiratory depression was detectable after treatment with antagonist, even in the entirely normal baby, it must be much more important to ensure adequate treatment in the asphyxiated, the preterm, or the otherwise at risk infant. The elimination-phase plasma half-life of naloxone after IV administration was about 3 hours, considerably longer than in the adult. Further studies are required to evaluate the most effective dosage, timing, and mode of administration.

We are indebted to Dr J. Thompson (Department of Physiology, University of Dundee) for his design and construction of the gas mixing chambers for the carbon dioxide output measurements and to Dr C. Ingram (Department of Physiology, University of St Andrews) for advice on the respiratory function tests. We thank Mr A. Whytock and Mr W. M. Tocher (Department of Child Health), Mr A. R. Rimmer (Department of Medical Physics), and Professor P. D. Griffiths and staff (Department of Biochemical Medicine), all of the University of Dundee, for technical assistance, Dr J. Bell and Dr S. Ackroyd for assessing the Scanlon scores, Mrs B. Forsyth and Miss L. Henderson for secretarial work, and all the nursing staff in the maternity department for their help. Dr G. W. Aherne (University of Surrey) kindly supplied the antiserum.

The work was supported by grants from the Wellcome Trust, Winthrop Laboratories, and the Tayside Area Health Board. 


\section{References}

Berkowitz, B. A., Ngai, S. H., Hempstead, J. , and Spector, S. (1975). Disposition of naloxone: use of a new radioiummunoassay. Journal of Pharmacology and Experimental Therapeutics, 195, 499-504.

Brackbill, Y., Kane, J., Manniello, R. L., and Abramson, D. (1974). Obstetric meperidine usage and assessment of neonatal status. Anesthesiology, 40, 116-121.

Bramwell, G. J., and Bradley, P. B. (1974). Actions and interactions of narcotic agonists and antagonists on brain stem neurones. Brain Research, 73, 167-170.

Brazelton, T. B. (1973). Neonatal Behavioural Assessment Scale. Clinics in Developmental Medicine No. 50. Heinemann Medical: London.

Cooper, L. V., Stephen, G. W., and Aggett, P. J. A. (1977). The climination of pethidine and bupivacaine in the newborn. Archives of Disease in Childhood, 52, 638-641.

Evans, J. M., Hogg, M. I. J., and Rosen, M. (1976). Reversal of narcotic depression in the neonate by naloxone. British Medical Journal, 2, 1098-1100.

Evans, J. M., Hogg, M. I. J., and Rosen, M. (1977). Measurement of carbon dioxide output, alveolar carbon dioxide concentration, and alveolar ventilation in the neonate. British Journal of Anaesthesia, 49, 453-456.

Gerhardt, T., Bancalari, E., Cohen, H., and Rocha, L. F. (1977). Use of naloxone to reverse narcotic respiratory depression in the newborn infant. Journal of Pediatrics, 90, 1009-1012,

Kappagoda, C. T., Stoker, J. B., and Linden, R. J. (1974). A method for the continuous measurement of oxygen consumption. Journal of Applied Physiology, 37, 604-607.
Moreland, T. A., Brice, J. E. H., and Walker, C. H. M. (1979). In press.

Nelson, N. M., Prod'hom, L. S., Cherry, R. B., Lipsitz, P. J., and Smith, C. A. (1962). Pulmonary function in the newborn infant. II. Perfusion. Estimation by analysis of the arterial-alveolar carbon dioxide difference. Pediatrics, 30, 975-989.

Newell, J. P., Kappagoda, C. T., and Linden, R. J. (1976). Method for continuous measurement of carbon dioxide output. Journal of Applied Physiology, 41, 810-813.

Ngai, S. H., Berkowitz, B. A., Yang, J. C., Hempstead, J., and Spector, S. (1976). Pharmacokinetics of naloxone in rats and in man: basis for its potency and short duration of action. Anesthesiology, 44, 398-401.

Scanlon, J. W., Brown, W. U., Weiss, J. B., and Alper, M. H. (1974). Neurobehavioural responses of newborn infants after maternal epidural anesthesia. Anesthesiology, 40, 121-128.

Thompson, J., Brice, J. E. H., and Walker, C. H. M. (1979). In press.

Wiener, P. C., Hogg, M. I. J., and Rosen, M. (1977). Effects of naloxone on pethidine-induced neonatal depression. I. Intravenous naloxone. British Medical Journal, 2, 228-231.

Correspondence to Dr J. E. H. Brice, Neonatal Unit, City Hospital, Hucknall Road, Nottingham NG5 1PB.

Received 26 September 1978 